Table S1 Details of all house mice obtained from Australia and the Netherlands subject to D-loop sequencing. Geographical coordinates are represented as decimal degrees. Most Australian samples were provided by Museum collections and the remaining samples belong to private collections of Michael Nachman, Kristin Ardlie [Ardlie KG, Silver LM (1998) Low frequency of t haplotypes in natural populations of house mice (Mus musculus domesticus). Evolution, 52, 1185-1196] and Michael Driessen. Samples provided by Michael Driessen were collected during pest management work of the Resource Management and Conservation Division, Department of Primary Industries, and Water, Tasmania, following their standard ethical practice. All Dutch samples were provided by Adri Rol at the Zoological Museum of Amsterdam. 'Sample ID’ corresponds to the original Museum Catalogue Number. 
Sample ID $\quad$ Sample provider $\quad$ Latitude Longitude $\quad$ Haplotype $\quad$ Clade AUSTRALIA
Western Australia

\begin{tabular}{|c|c|c|c|c|c|}
\hline 36261 & WAM & $-31,2300$ & 116,3017 & AUSTRALIA.01 & Clade E \\
\hline 49891 & WAM & $-31,2300$ & 116,3017 & AUSTRALIA.01 & Clade E \\
\hline 44110 & WAM & $-28,2000$ & 123,6000 & AUSTRALIA.02 & Clade E \\
\hline 44124 & WAM & $-28,2000$ & 123,6000 & AUSTRALIA.07 & Clade F \\
\hline 56193 & WAM & $-26,9397$ & 120,5581 & AUSTRALIA.01 & Clade E \\
\hline 48520 & WAM & $-25,0972$ & 118,0083 & AUSTRALIA.01 & Clade E \\
\hline 49678 & WAM & $-29,2822$ & 117,5742 & AUSTRALIA.01 & Clade E \\
\hline 49687 & WAM & $-29,2819$ & 117,6428 & AUSTRALIA.05 & Clade F \\
\hline 56476 & WAM & $-34,2214$ & 116,3741 & AUSTRALIA.01 & Clade E \\
\hline 56486 & WAM & $-34,0878$ & 116,3194 & AUSTRALIA.13 & Clade B \\
\hline АВТС07343 & SAM & $-30,8579$ & 128,1011 & AUSTRALIA.05 & Clade F \\
\hline АВТС07352 & SAM & $-30,8579$ & 128,1011 & AUSTRALIA.05 & Clade F \\
\hline АВТС07414 & SAM & $-33,4623$ & 123,8421 & AUSTRALIA.05 & Clade F \\
\hline ABTC07415 & SAM & $-33,4623$ & 123,8421 & AUSTRALIA.05 & Clade F \\
\hline ABTC07417 & SAM & $-33,4623$ & 123,8421 & AUSTRALIA.05 & Clade F \\
\hline АВТС07421 & SAM & $-31,8341$ & 128,1776 & AUSTRALIA.05 & Clade F \\
\hline АВТС63123 & SAM & $-21,3031$ & 118,8611 & AUSTRALIA.01 & Clade E \\
\hline \multicolumn{6}{|c|}{ Northern Territory } \\
\hline ABTC41764 & SAM & $-25,5900$ & 129,2858 & AUSTRALIA.05 & Clade F \\
\hline АВТС24032 & SAM & $-23,7354$ & 133,3307 & AUSTRALIA.05 & Clade F \\
\hline АВТС24033 & SAM & $-22,7354$ & 133,3307 & AUSTRALIA.05 & Clade F \\
\hline ABTC28266 & SAM & $-12,1920$ & 136,7742 & AUSTRALIA.03 & Clade E \\
\hline M30774 & ANWC & $-12,7617$ & 133,1033 & AUSTRALIA.06 & Clade F \\
\hline \multicolumn{6}{|l|}{ South Australia } \\
\hline ABTC37735 & SAM & $-37,0342$ & 139,5117 & AUSTRALIA.05 & Clade F \\
\hline ABTC37731 & SAM & $-37,1336$ & 139,4804 & AUSTRALIA.05 & Clade F \\
\hline АВТС37481 & SAM & $-37,0812$ & 140,4142 & AUSTRALIA.05 & Clade F \\
\hline АВТС37501 & SAM & $-37,5336$ & 140,2228 & AUSTRALIA.05 & Clade F \\
\hline АВТС37430 & SAM & $-38,0315$ & 140,5635 & AUSTRALIA.01 & Clade E \\
\hline ABTC26669 & SAM & $-32,0842$ & 134,4352 & AUSTRALIA.05 & Clade F \\
\hline АВТС26670 & SAM & $-32,0751$ & 134,3943 & AUSTRALIA.05 & Clade F \\
\hline ABTC26679 & SAM & $-31,4619$ & 133,2820 & AUSTRALIA.05 & Clade F \\
\hline ABTC26682 & SAM & $-31,4742$ & 133,2545 & AUSTRALIA.05 & Clade F \\
\hline АВТС35543 & SAM & $-29,3615$ & 135,4335 & AUSTRALIA.05 & Clade F \\
\hline АВТС35714 & SAM & $-29,1721$ & 135,1100 & AUSTRALIA.05 & Clade F \\
\hline ABTC35426 & SAM & $-29,0324$ & 136,1556 & AUSTRALIA.02 & Clade E \\
\hline АВТС36313 & SAM & $-28,5703$ & 136,4655 & AUSTRALIA.05 & Clade F \\
\hline АВТС36796 & SAM & $-31,2517$ & 140,1515 & AUSTRALIA.05 & Clade F \\
\hline АВТС36799 & SAM & $-31,2617$ & 140,2807 & AUSTRALIA.05 & Clade F \\
\hline АВТС36797 & SAM & $-31,2438$ & 140,1035 & AUSTRALIA.05 & Clade F \\
\hline АВТС36633 & SAM & $-30,2557$ & 140,5826 & AUSTRALIA.05 & Clade F \\
\hline
\end{tabular}


Sample ID Sample provider Latitude Longitude $\quad$ Haplotype Clade

South Australia (cont.)

$\begin{array}{llllll}\text { ABTC79657 } & \text { SAM } & -34,6004 & 138,7491 & \text { AUSTRALIA.11 } & \text { Basal } \\ \text { ABTC33350 } & \text { SAM } & -35,8389 & 137,1679 & \text { AUSTRALIA.11 } & \text { Basal } \\ \text { ABTC33353 } & \text { SAM } & -35,8389 & 137,1679 & \text { AUSTRALIA.11 } & \text { Basal } \\ \text { ABTC33534 } & \text { SAM } & -35,8389 & 137,1679 & \text { AUSTRALIA.11 } & \text { Basal } \\ \text { ABTC33610 } & \text { SAM } & -35,8389 & 137,1679 & \text { AUSTRALIA.11 } & \text { Basal } \\ \text { ABTC33650 } & \text { SAM } & -35,8389 & 137,1679 & \text { AUSTRALIA.11 } & \text { Basal }\end{array}$

Queensland

\begin{tabular}{|c|c|c|c|c|c|}
\hline KA107 & MN/KA & $-27,5000$ & 150,5000 & AUSTRALIA.08 & Clade D \\
\hline KA112 & $\mathrm{MN} / \mathrm{KA}$ & $-27,5000$ & 150,5000 & AUSTRALIA.08 & Clade D \\
\hline KA124aust & $\mathrm{MN} / \mathrm{KA}$ & $-27,5000$ & 150,5000 & AUSTRALIA.10 & Clade D \\
\hline KA1aust & $\mathrm{MN} / \mathrm{KA}$ & $-27,5000$ & 150,5000 & AUSTRALIA.08 & Clade D \\
\hline KA29aust & $\mathrm{MN} / \mathrm{KA}$ & $-27,5000$ & 150,5000 & AUSTRALIA.09 & Clade D \\
\hline KA35aust9205 & $\mathrm{MN} / \mathrm{KA}$ & $-27,5000$ & 150,5000 & AUSTRALIA.08 & Clade D \\
\hline KA41aust & $\mathrm{MN} / \mathrm{KA}$ & $-27,5000$ & 150,5000 & AUSTRALIA.08 & Clade D \\
\hline KA9 & $\mathrm{MN} / \mathrm{KA}$ & $-27,5000$ & 150,5000 & AUSTRALIA.08 & Clade D \\
\hline ABTC13890 & SAM & $-25,5023$ & 138,4104 & AUSTRALIA.02 & Clade E \\
\hline АВTC79716 & SAM & $-26,6422$ & 149,6230 & AUSTRALIA.02 & Clade E \\
\hline M30797 & ANWC & $-27,4900$ & 152,8897 & AUSTRALIA.08 & Clade D \\
\hline \multicolumn{6}{|l|}{ New South Wales } \\
\hline KA35aust9204 & MN/KA & $-34,8058$ & 145,8841 & AUSTRALIA.04 & Clade E \\
\hline KA54 & $\mathrm{MN} / \mathrm{KA}$ & $-34,8058$ & 145,8841 & AUSTRALIA.01 & Clade E \\
\hline M29998 & ANWC & $-35,5400$ & 149,2100 & AUSTRALIA.01 & Clade E \\
\hline \multicolumn{6}{|c|}{ Australia Capital Territory } \\
\hline M30792 & ANWC & $-35,2192$ & 149,1033 & AUSTRALIA.01 & Clade E \\
\hline M30794 & ANWC & $-35,2192$ & 149,1033 & AUSTRALIA.01 & Clade E \\
\hline M30795 & ANWC & $-35,2192$ & 149,1033 & AUSTRALIA.01 & Clade E \\
\hline M30796 & ANWC & $-35,2192$ & 149,1033 & AUSTRALIA.01 & Clade E \\
\hline \multicolumn{6}{|l|}{ Tasmania } \\
\hline A410 & TMAG & $-42,8817$ & 147,3322 & AUSTRALIA.01 & Clade E \\
\hline A504 & TMAG & $-42,9129$ & 147,3545 & AUSTRALIA.01 & Clade E \\
\hline A505 & TMAG & $-42,9129$ & 147,3545 & AUSTRALIA.01 & Clade E \\
\hline A1481a & TMAG & $-42,8795$ & 147,3275 & AUSTRALIA.12 & Basal \\
\hline A1481b & TMAG & $-42,8795$ & 147,3275 & AUSTRALIA.12 & Basal \\
\hline A1575 & TMAG & $-42,8731$ & 147,3023 & AUSTRALIA.01 & Clade E \\
\hline A3233 & TMAG & $-43,0624$ & 147,8123 & AUSTRALIA.01 & Clade E \\
\hline TAS1 & MD & $-42,9796$ & 147,3262 & AUSTRALIA.01 & Clade E \\
\hline TAS2 & MD & $-42,9796$ & 147,3262 & AUSTRALIA.01 & Clade E \\
\hline TAS3 & MD & $-42,9796$ & 147,3262 & AUSTRALIA.01 & Clade E \\
\hline TAS4 & MD & $-42,9796$ & 147,3262 & AUSTRALIA.01 & Clade E \\
\hline TAS5 & MD & $-42,9796$ & 147,3262 & AUSTRALIA.01 & Clade E \\
\hline TAS6 & MD & $-42,9796$ & 147,3262 & AUSTRALIA.01 & Clade E \\
\hline TAS7 & MD & $-42,9796$ & 147,3262 & AUSTRALIA.01 & Clade E \\
\hline
\end{tabular}




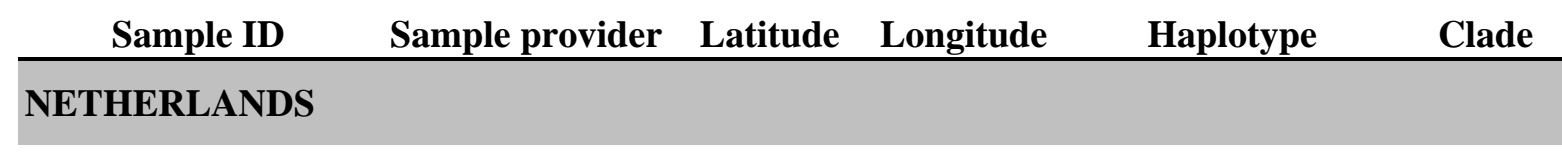

Rotterdam

$\begin{array}{llllll}5902 & \text { ZMA } & 51,9226 & 4,4707 & \text { NETHERLANDS.04 } & \text { Clade E } \\ 5897 & \text { ZMA } & 51,9226 & 4,4707 & \text { NETHERLANDS.05 } & \text { Clade C } \\ 5898 & \text { ZMA } & 51,9226 & 4,4707 & \text { NETHERLANDS.04 } & \text { Clade E }\end{array}$

Oosthuizen

$8139 \quad$ ZMA $\quad 52,5747 \quad 4,9985 \quad$ NETHERLANDS.06 $\quad$ Clade D

Midsland noord

$27479 \quad$ ZMA $\quad 53,3631 \quad 5,2110 \quad$ NETHERLANDS.05 Clade C

Terschelling

\begin{tabular}{|c|c|c|c|c|}
\hline 27476 & ZMA & 53,3631 & 5,2110 & NETHERLANDS.01 \\
\hline 27477 & ZMA & 53,3631 & 5,2110 & NETHERLANDS.01 \\
\hline
\end{tabular}

Montfort

$4674 \quad$ ZMA $51,1173 \quad 5,9521 \quad$ NETHERLANDS.01 $\quad$ Clade E

Maastricht

\begin{tabular}{cccccc}
\hline \multicolumn{1}{c}{4393} & ZMA & 50,8498 & 5,6873 & NETHERLANDS.07 & Clade D \\
Geulle & & & & & \\
\hline Breda & ZMA & 50,9225 & 5,7484 & NETHERLANDS.03 & Clade E \\
\hline 5369 & & & & & \\
\hline 5368 & ZMA & 51,5893 & 4,7745 & NETHERLANDS.01 & Clade E \\
Groenekan & ZMA & 51,5893 & 4,7745 & NETHERLANDS.01 & Clade E \\
\hline 5949 & & & & & \\
\hline Amsterdam & ZMA & 52,1229 & 5,1519 & NETHERLANDS.02 & Clade E \\
\hline 4401 & & & & & \\
4669 & ZMA & 52,3739 & 4,8941 & NETHERLANDS.01 & Clade E \\
11604 & ZMA & 52,3739 & 4,8941 & NETHERLANDS.05 & Clade C \\
& ZMA & 52,3739 & 4,8941 & NETHERLANDS.05 & Clade C \\
& ZMA & 52,3739 & 4,8941 & NETHERLANDS.03 & Clade E
\end{tabular}

\section{Sample providers}

WAM - Western Australia Museum

SAM - South Australian Museum

ANWC - Australia National Wildlife Collection

TMAG - Tasmanian Museum and Art Gallery

ZMA - Zoological Museum of Amsterdam

MN/KA - Michael Nachman / Kristin Ardlie

MD - Michael Driessen 